\title{
Study on the Super-resolution Reconstruction Algorithm for Remote Sensing Image Based on Compressed Sensing
}

\author{
Qiang Yang ${ }^{1,2, a}$, HuaJun Wang ${ }^{1, b}$ and Xuegang Luo ${ }^{1, \mathrm{c}}$ \\ 1. College of Geophysical, ChengDu University of Technology, China \\ 2. College of Computer and Information Engineering, Yibin University, China \\ a.scyangqiang@163.com,b.63918058@qq.com,c.543884841@qq.com
}

\begin{abstract}
Image super resolution reconstruction has important significance in remote sensing image feature extraction and classification etc.. Because the remote sensing image size is larger, it is difficult to super resolution reconstruction using multiple images, the compressed sensing (CS) theory was introduced into the super-resolution reconstruction. Algorithm designed the low pass filter to reduce the sample correlation matrix and wavelet, at the same time, the algorithm selects the partial Hadamard-matrix as the measurement matrix, it has faster reconstruction speed and low storage requirements, which ensure that the image reconstruction keep with the RIP criterion of compressed sensing theory. Finally, this paper realizes the remote sensing image super resolution reconstruction through the improved iterative algorithm. Experiments show that the reconstructed images of the PSNR value has increased, the reconstructed image has a better visual effect.
\end{abstract}

Keywords: Compressed Sensing, Super-Resolution Reconstruction, Remote Sensing Image, Image Reconstruct Technique

\section{Introduction}

At present, remote sensing image has been used in resource surveys, marine monitoring, environmental monitoring, geological survey and other fields. With the continuous increase in the number of satellites, and greatly improve the manufacturing level of satellite sensors, the remote sensing image data available to people increase sharply. Remote sensing image meet the requirements of the application, at the same time, the remote sensing image also has a problem in image quality degradation and fuzzy, because satellite high speed flight and atmospheric turbulence disturbance. At the same time, due to the limited funds and hardware conditions and other factors, remote sensing images are mostly low resolution. Even the remote sensing image obtained from the IKONOS-QuicBird satellite, their resolution is about $0.5 \mathrm{~m}$ [1]. For the current remote sensing image analysis, object recognition and feature extraction, people need better quality and higher resolution. From hardware to improve the resolution of remote sensing images still exist funds and technical limitations. Therefore, super-resolution reconstruction is performed using available remote sensing image, so as to obtain higher resolution remote sensing images has important significance.

Super resolution image reconstruction mainly includes two ways, one is using multiple low resolution images from different time, different angles or different sensors, using complementary information of multiple images, the algorithm for the reconstruction of the right to obtain a high resolution image, the other way is direct reconstruction using a single low resolution to obtain a high resolution image [2]. In the last century 60's, Harris [3] and Goodman [4] first proposed the concept of optical image super resolution reconstruction. In 1995, Hunt [5] first analyzes the possibility of super-resolution reconstruction theory. At present, the super-resolution image reconstruction algorithm is 
divided into two categories: Based on the reconstruction method and based on learning method.

The algorithm based on reconstruction hypothesis is that the high resolution image can get low resolution images, and reconstructed image through the acquisition process of image modeling fuzzy and down sampling. The algorithm based on reconstruction can be divided into reconstruction algorithm based on space-domain and reconstruction algorithm based on frequency-domain. The reconstruction algorithm based on space-domain included projection onto convex sets (POCS), iterative back projection method (IBP), sample interpolation method (Non-uniform, Interpolation), the maximum likelihood estimation method (ML), maximum a posteriori-estimation (MAP), and the hybrid MAP/POCS method. The algorithm reconstruction in frequency domain included aliasing reconstruction method based on Fu-Liye transform, and reconstruction algorithm based on wavelet or DCT transform.

The reconstruction algorithm based on learning is a method of recognition based on prior knowledge. The reconstruction algorithm based on learning included: learning method based on a priori-operator identification, the reconstruction algorithms based on priori-model of texture, the reconstruction algorithm based on vector quantization of training sample tissue, the reconstruction method based on the theory of multi-scale tensor voting, and the blind super-resolution reconstruction method by estimating the PSF parameters of pixel sensors [6].

The super-resolution image reconstruction algorithm often requires multi-frame image to obtain better reconstruction results [7]. However, the scale of remote sensing image is larger, the super - resolution reconstruction faced the real-time processing problem of mass data image using multiple images.

This paper presents a single image super-resolution reconstruction based on compressed sensing. The reconstruction algorithm of compressed sensing introduced the low pass filter, the algorithm reduces the correlation of sample matrix and wavelet, at the same time, the algorithm uses local Hadamard-matrix as the measurement matrix, it has faster reconstruction speed and low storage space requirement, which ensures that the image reconstruction algorithm of compressed sensing meets RIP criterion. Finally, this paper realizes the super resolution reconstruction of remote sensing image by the improved iterative algorithm.

\section{The Compressed Sensing Theory}

In 2006, Candès, Donoho and Tao et al proposed compressed sensing (CS) theory [8-10], they proved that the signal can be reduced dimension measurement to obtain a small amount of data through the measurement matrix, the signal can be sparse representation in a transform matrix. At the same time, we can efficiently reconstruct the original signal using nonlinear optimization algorithm to reduce the dimensionality of the measurement data.

Let $f$ is one-dimensional discrete signal, the length is $\mathrm{N}, \Phi$ is a set of orthogonal basis vectors, $u$ is the K-sparse signal, $\varepsilon$ is the minimum error value, if the original signal $f$ can be expressed by equation (1), the signal is $\mathrm{K}$-sparse.

$$
f=\Phi u+\varepsilon
$$

In the formula (1), $u \in R^{N}$, $\mathrm{u}$ is the only $\mathrm{K}$ non-zero value of the one-dimensional vector $(\mathrm{K}<<\mathrm{N})$.

The algorithm makes the reduce-dimension measurement for K-sparse signal, Measurement formula as shown in formula 2:

$$
x=\Phi \Psi^{-1} \Psi u+\varepsilon=\tilde{\Phi} \theta+\varepsilon=\Theta u+\varepsilon
$$


In the formula (2), $\theta=\Psi u, \tilde{\Phi}=\Phi \Psi^{-1}, \Psi$ represents the orthogonal transform, such as curvelet transform, wavelet transform, triangle transform, $\Psi^{-1}$ is the contrary transform, $\Theta$ is the measurement matrix $(M \times N), \Theta=\Phi \Psi^{-1} \Psi, x$ is the length dimension reduction for $\mathrm{M}$ measurements $(\mathrm{M}<\mathrm{N})$.

$$
1-\partial_{k} \leq \frac{\|\Theta f\|_{2}^{2}}{\|f\|_{2}^{2}} \leq 1+\partial_{k}
$$

In the formula $3, \partial_{k}$ is a constant between 0 and $1, \partial_{k} \in(0,1), \quad$ so we can reconstruct the original signal $f$ with value $x$ of measurements.

D. Donoho [8] proved that the algorithm can use the minimum $l_{1}$-norm reconstructs the source K- sparse signals with high probability.

$$
\hat{f}=\arg \min \left\|f^{\prime}\right\|_{1} \quad \text { s.t } \quad \Theta f^{\prime}=x
$$

In the formula (4), $\hat{f}$ is the signal reconstruction, $x$ is the observation vector. Signal reconstruction is a convex optimization problem with a solution of minimum $l_{1}$-norm, can be used the algorithm of the matching pursuit (MP) to achieve, the matching pursuit (MP) basic idea is through the $\mathrm{N}$ iteration, select the best matched atoms from the measurement matrix $\Phi$, the atoms is the best matching to the observation signal margin.

The basic principle of matching pursuit (MP) algorithm is:

Assume that $n=1, \hat{f}^{0}=0$, the candidate subset $\Gamma^{0}$ is empty, the observation signal margin $r^{0}$ is the initial observation signal $\mathrm{x}$.

Firstly, using the equation 5 to obtain the best matching atom $I^{n}$ :

$$
I^{n}=\arg \max _{i=1,2 \ldots N}<r^{n-1}, \Phi_{i}>
$$

Then, add the best matching atom $I^{n}$ to the candidate subset of $\Gamma$. To solve the update candidate subset of $\Gamma$ :

$$
\Gamma^{n}=\Gamma^{n-1} \cup I^{n}
$$

And then solve the estimation signal $\hat{f}^{n}=\Phi_{\Gamma n}^{+} x . \Phi_{\Gamma n}^{+}$is the $\Phi_{\Gamma n}$ pseudo inverse of Moore-Penrose[11] .Then, to solve the observation signal margin $r^{n}, r^{n}=x-\Phi_{\Gamma n} \hat{f}^{n}$ 。

Repeat the calculation of $\hat{f}$ and $\mathrm{r}$, when $r=\sigma^{2}$ end of iteration ( $\sigma^{2}$ is the noise variance).

\section{The Super Resolution Reconstruction Algorithm based on Compressed Sensing}

CS theory breaks through the traditional Nyquist-sampling theorem, it samples the signal much less than the Nyquist-sampling rate. CS theory reduces the signal sampling rate, greatly reducing the data transmission, processing and storage requirements. CS theory provides a new idea for the super resolution reconstruction of the large data on remote sensing image.

In this paper, the compressed sensing (CS) theory was introduced into the super-resolution reconstruction. Algorithm designed the low pass filter to reduce the sample correlation matrix and wavelet, at the same time, the algorithm selects the partial Hadamard-matrix as the measurement matrix, it has faster reconstruction speed and low 
storage requirements, which ensure that the image reconstruction keep with the RIP criterion of the compressed sensing theory. Finally, this paper realizes the remote sensing image super resolution reconstruction through the improved iterative algorithm.

\subsection{The Construction of Learning Dictionary}

In order to improve the effect of image super-resolution reconstruction, this paper constructs the learning dictionary. Firstly, the algorithm use the block feature of high resolution image and low resolution image for training, and obtain the high resolution dictionary and low resolution dictionary with sparse representation.

$D_{l}$ is the low resolution dictionary, the objective function is defined as:

$$
D_{l}, Z=\underset{D l, Z}{\arg \min }\left\|Y-D_{l} Z\right\|_{F}^{2} \text { s.t. }\left\|z_{i}\right\| 0 \leq T
$$

In the formula $7, \mathrm{Z}$ is the low resolution remote sensing image feature sparse representation coefficients on low resolution dictionary. When the high resolution dictionary has the same sparse representation $\mathrm{Z}$ as the low resolution dictionary, high resolution dictionary learning is defined as:

$$
D_{h}=\underset{D h}{\arg \min }\left\|x_{h}-x_{l}-\left[\sum_{k \in \Omega} R_{k}{ }^{T} R_{k}\right]^{-1}\left[\sum_{k \in \Omega} R^{T}{ }_{k} D_{h} z_{k}\right]\right\|_{2}^{2}
$$

In the formula $8, R_{k}$ is the parameters of extraction operator, $R_{k}^{T} D_{h} z_{k}$ is the position of reconstructed images in high resolution images [17].

\subsection{The Algorithm of Super-resolution Reconstruction Based on CS}

Let $\mathrm{F}$ represent the original low resolution image, $\mathrm{X}$ is the high resolution image, the observation model of super resolution reconstructions for remote sensing image as shown in formula 9:

$$
F=D H X+v
$$

In the formula $9, \mathrm{D}$ is the sub sampling matrix, $\mathrm{H}$ is the fuzzy matrix, $\mathrm{V}$ is adding Gauss noise.

In order to reduce the correlation between sample matrix and wavelet, the algorithm introduce a low-pass filter in the super resolution reconstruction, the low-pass filter is the product of filter matrix $G$ and the transformation matrix [14], $\Phi=\phi^{H} G \phi$. If the high resolution image $\mathrm{X}$ can be sparse representation, as $a_{X}=\Psi^{-1} X,\left(\Psi^{-1}\right.$ is a sparse transform, $\Psi$ is a sparse inversion transform). The algorithm uses CS theory sampling for low resolution images $\mathrm{F}$, and obtain a new signal $\mathrm{f}$, the calculate formula of $\mathrm{f}$ as shown in formula 10[12]:

$$
f=\Phi F=\Phi D H X+v=\tilde{\Phi} X+v=\tilde{\Phi} \Psi a_{X}+v=\Theta a_{X}+v
$$

In the formula $10, \tilde{\Phi}=\Phi D H, \Theta=\tilde{\Phi} \Psi$, If $\Theta$ satisfies the RIP criterion, $\tilde{\Phi}$ is not related to $\Psi$, according to the theory of compressed sensing, we can reconstruct a high resolution image $\hat{X}$ :

$$
\hat{X}=\arg \min _{X}\left\|\Psi^{-1} X\right\|_{1} \quad \text { s.b. } \quad\|f-\tilde{\Phi} X\|_{2} \leq \varepsilon
$$

In order to ensure that $\Theta$ satisfies RIP criterion, this paper selects the partial Hadamard matrix as the measurement matrix, partial Hadamard matrix has faster reconstruction speed and low storage requirements [13], the formula of partial Hadamard matrix as shown in formula 12: 


$$
\phi=Q_{M} W_{B} P_{N}
$$

In the formula, $12, Q_{M}$ is the operator parameters, $W_{B}$ is a diagonal matrix, each sub block is a Hadamard matrix, $P_{N}$ is the random scrambling matrix, the input scrambling signal ensure that $\phi$ is not correlation with the original signal. The partial Hadamard matrix is randomly selected $\mathrm{M}$ lines from the Hadamard matrix of $\mathrm{N}$ dimension.

Because of the $P_{N}$ scrambling, the image after D-sampling is not correlation with $\phi$. Fuzzy matrix $\mathrm{H}$ make convolution of the original image, the literature [15] has been demonstrated that $\mathrm{H}$ operation can increase the uncorrelated $\phi$ and $\mathrm{D}$.Therefore, $\Theta=\Phi D H \Psi$ satisfies RIP condition.

Super resolution image reconstruction is different from the ordinary reconstruction of compressed sensing image. Based on observation model of super resolution reconstruction and dictionary learning, the matching pursuit algorithm is improved. The improved algorithm of super resolution reconstruction based on compressed sensing is as follows:

Step 1: Input the high resolution dictionary $D_{h}$ and low resolution dictionary $D_{l}$, and input the low resolution remote sensing image $F$.

Step 2: Initialized the number of iterations (n) to 1, the results of reconstruction image $(\hat{X})$ be initialized to $0, \hat{X}=0$.

Step 3: Based on the degradation model $F=D H X+v$, the algorithm make the projection observation for the low resolution images $f=\Phi F=\tilde{\Phi} X$.

Step 4: According to the iterative algorithm for solving $X_{n+1}$ :

$$
X_{n+1}=\Psi\left(\Gamma_{T}\left(\Psi^{-1}\left(X_{n}+\tilde{\Phi}^{-1}\left(f-\tilde{\Phi} X_{n}\right)\right)\right)\right)
$$

In the formula $13, \Gamma_{T}(x)=\left\{\begin{array}{l}x,|x| \geq T \\ 0,|x|<T\end{array}, \quad \tilde{\Phi}^{-1}=P^{-1} D^{-1} \Phi^{-1}\right.$, among them, $P^{-1}$ is the deblurring operator, $\Phi^{-1}$ is the inversion of partial Hadamard matrix [16].

Step 5: Computing the sparse representation coefficients $a^{\prime}$ of low resolution feature of $\mathrm{g}$ on low resolution dictionary $D_{l}$, and obtain the high resolution image based on the sparse coefficient $a^{\prime}$ and the high resolution dictionary $D_{h}$.

$$
a^{\prime}=\arg \min \left\|g-D_{l} a\right\|_{2}^{2}+\left\|Z D_{h} a-w\right\|_{2}^{2}+\lambda\|a\|_{1}
$$

Step 6: Update the reconstructed image subsets, $\hat{X}=\hat{X}+X_{n+1}$, and the number of iteration(n) plus $1, \mathrm{n}=\mathrm{n}+1$.

Step 7: Calculating the observed signal margin (residual) $\gamma, \gamma=f-\hat{\Phi} \hat{X}$.

Step 8: Repeated the steps of 3 to 7 , when $\gamma=\sigma^{2}$, complete the iterative reconstruction.

\section{The Experimental Analysis}

The experiment selected the remote sensing images from the "geo spatial data cloud" (http://www.gscloud.cn/) as experimental data. The experiment captures the remote sensing image to keep the image size of $256 * 256$ pixel resolution. And degrade the remote sensing image to the resolution of $128 * 128$. This paper adopts bilinear interpolation method, the algorithm of super resolution reconstruction based on sparse representation (SRSR) and the methods in this paper for reconstruction experiment. 
The experimental results as shown in Figure 1. Figure 1 (a) is the degraded image $128 * 128$,Figure 1 (b) is the high resolution raw image for reference, figure 1 (c) is the bilinear interpolation reconstruction results, Fig. 1 (d) is the reconstruction of the SRSR method, Figure 1 (e) is the reconstruction effect of this paper.

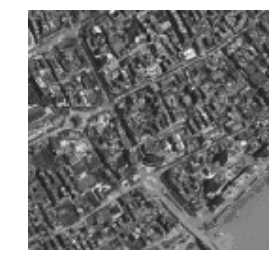

Figure 1 (a).The Degraded Image 128*128

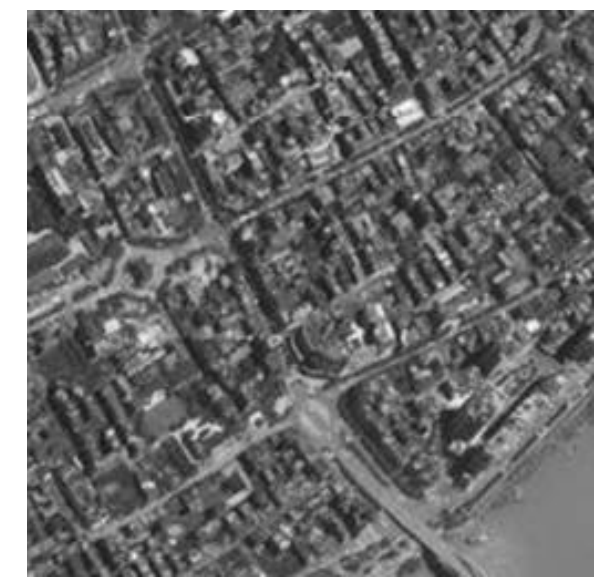

Figure 1 (b). The High Resolution Raw Image for Reference

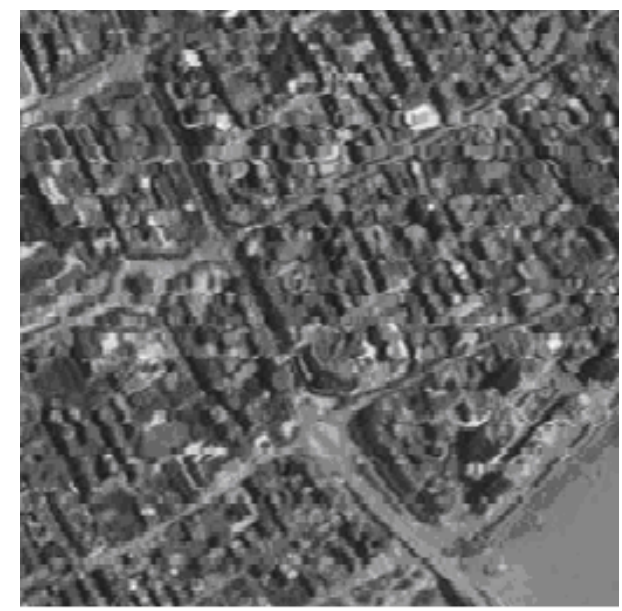

Figure $1(d)$. The

Reconstruction of the SRSR Method

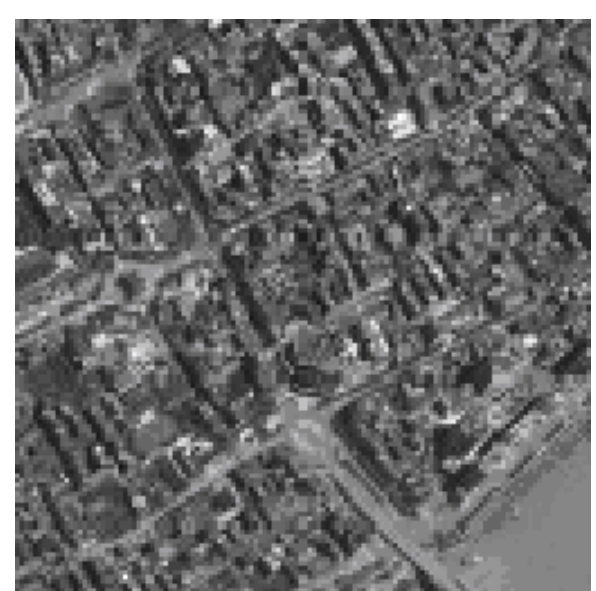

Figure 1 (c). The Bilinear Interpolation Reconstruction Results

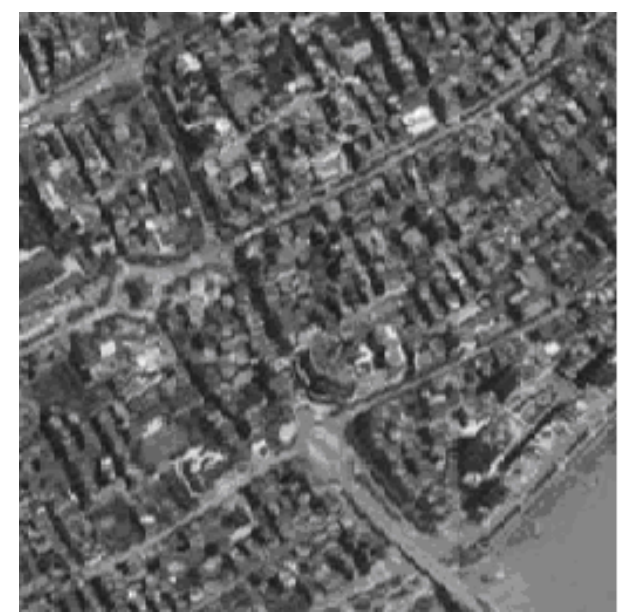

Figure 1 (e).The Reconstruction Effect of this Paper

\section{Figure 1. The Experimental Results with Different Super-resolution Reconstruction Algorithm}

From the analysis of visual effect, high resolution image reconstruction of SRSR algorithm and the algorithm of this paper have better visual effect, image quality is significantly higher than the low resolution image quality degradation. 
At the same time, we make the quantitative analysis and comparison of the various algorithms of remote sensing image super resolution reconstruction results using the peak signal-to-noise ratio (PSNR) in this paper.

Figure 1 (c), the PSNR value of bilinear interpolation for image reconstruction is 26.21, Figure 1 (d), the PSNR value of SRSR algorithm for image reconstruction is 29.36, Figure 1 (E), the PSNR value of reconstruction algorithm in this paper is 29.76. The higher the PSNR value, the better the effect of super resolution image reconstruction.

In order to further test this algorithm reconstruction effect, this paper makes 2 times, 4 times and 8 times of the degraded image super-resolution reconstruction test, the PSNR value of different magnifications image reconstruction as shown in Table 1:

\section{Table 1. The PSNR Value of Different Magnifications Image Reconstruction}

\begin{tabular}{|c|c|c|c|}
\hline \multirow{2}{*}{ algorithm } & \multicolumn{3}{|c|}{$\begin{array}{l}\text { the PSNR value of different magnifications } \\
\text { image reconstruction }\end{array}$} \\
\hline & 2 times & 4 times & 8 times \\
\hline bilinear interpolation & 26.21 & 22.10 & 18.52 \\
\hline SRSR algorithm & 29.36 & 23.49 & 20.15 \\
\hline $\begin{array}{l}\text { reconstruction algorithm } \\
\text { in this paper }\end{array}$ & 29.76 & 23.60 & 20.18 \\
\hline
\end{tabular}

The data in Table 1 shows that, the greater the magnification, the PSNR value is smaller, the worse the effect of image reconstruction. Experiments show that, the super resolution reconstruction algorithm of remote sensing image based on CS achieved better effect.

\section{Summary}

Compressed sensing theory is widely used in image processing, the compressed sensing (CS) theory was introduced into the super-resolution reconstruction, this paper implements super-resolution reconstruction based on compressed sensing. In order to reduce the sample correlation matrix and wavelet matrix, the low-pass filter is introduced in the algorithm of super resolution reconstruction. At the same time, the algorithm selects the partial Hadamard as measurement matrix, the matrix has faster reconstruction speed and low storage space requirement, which ensure that the image reconstruction keep with RIP criterion of compressed sensing theory.

Experiments show that, the reconstruction image has a better visual effect, the algorithm PSNR is better than that of the bilinear interpolation and SRSR algorithm. Experiments show that the algorithm achieved better reconstruction effect, which provides a new idea algorithm for remote sensing image super resolution reconstruction.

\section{Acknowledgements}

This work was financially supported by the Research Foundation of Department of Education in Sichuan Province (NO: 13ZB0212), The key technology projects research foundation of Yibin City (NO: 2012SF016), and the Research Foundation of Yibin University (NO: 2012S09).

\section{References}

[1] Y. Shi, W. Wu and D. Luo, "Contourlet domain of image super-resolution restoration technique based on remote sensing [J]", computer application, vol. 30, no. 4, (2010), pp. 939-942.

[2] L. Xiao and Z. Wei, "Super resolution reconstruction of image non local regularization model and algorithm [J]", Chinese Journal of computers, vol. 34, no. 5, (2011), pp. 931-941. 
[3] J. L. Harri, "Diffraction and resolving power [J]", Journal of the Optical Society of America, vol. 54, no. 7, (1964), pp. 931一936.

[4] J. W. Goodman, "Introduction to Fourier optics [M]", McGraw-Hill, NewYork, (1968).

[5] B. R. Hunt, "Super-resolution of images: algorithms, Principles, performance [J]", International Journal of Imaging Systems and Technology, vol. 6, (1995), pp. 297-304.

[6] J. Qiao, "Enhancement technology research on super resolution reconstruction and image [D]", Shandong: Shandong University, (2008), pp. 28-48.

[7] Y. Zhang, K. Xu and Y. Li, "Super resolution of remote sensing image based on storage and POCS method [J]", Journal of Tsinghua University (NATURAL SCIENCE EDITION), vol. 50, no. 10, (2010), pp. 1743-1747.

[8] D. Donoho, "Compressed sensing [J]", IEEE Trans. Info. Theory, vol. 52, no. 4, (2006), pp. 1289-1306.

[9] E. Candès, J. Romberg and T. Tao, "Robust uncertainty principles: Exact signal recognition from highly incomplete frequency information [J]”, IEEE Trans. Info. Theory, vol. 52, no. 2, (2006), pp. 489-509.

[10] E. Candès and T. Tao, "Near optimal signal recovery from random projections: Universal encoding strategies [J]”, IEEE Trans. Info. Theory, vol. 52, no. 12, (2006), pp. 5406-5425.

[11] Z. Li, I"mage reconstruction algorithm based on compressed sensing [D]", Beijing: Beijing Jiaotong University, (2012), pp. 26-36.

[12] P. Zhang, "Research on the image super resolution that can realize the self-repairing of compressed sensing [D]", Qinhuangdao: Yanshan University, (2013), pp. 70-100.

[13] T. T. Do, T. D. Tran and G. Lu, "Fast compressive sampling with structurally random matrices [C]", IEEE International Conference on Acoustics Speech and Signal Processing, vol. 4, (2008), pp. 3369-3372.

[14] P. Sen and S. Darabi, "Compressive image super-resolution [C]", 43rd Asilomar Conference on Signals, Systems and Computers, (2009).

[15] E. Candes, Y. Eldar and D. Needell, "Compressed sensing with coherent and redundant dictionaries [J]", Appl. Comput. Harmon. Anal, vol. 31, (2013), pp. 59-73.

[16] P. Zhang and H. Zhang, "Research on image super resolution that can realize the self-repairing of compressed sensing [J]", Journal of Computer Aided Design \& computer graphics, vol. 24, no. 12, (2012), pp. 1621-1631.

[17] J. Zhong, "Research on the super resolution reconstruction algorithm of optical remote sensing image based on sparse representation [D]", Nanjing: Nanjing Normal University, (2013), pp. 65-75.

\section{Authors}

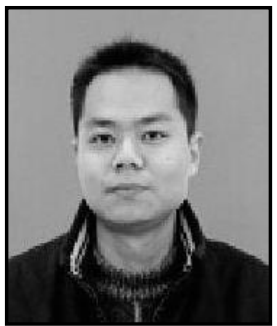

Qiang Yang, he is Associate professor. He graduated from the Chengdu University of Technology and received a master's degree in 2007. In 2013, He went to Germany to study at Rheinisch Westfaelische Technische Hochschule Aachen University. Now, he is the doctoral student at Chengdu University of Technology. His main research direction is image processing and pattern recognition.

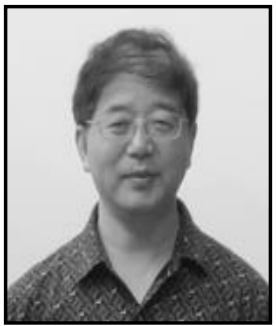

Huajun Wang, he is $\mathrm{Ph}$. D and Professor, He is the Sichuan Province Science and technology leader. In 2001, he graduated from the University of Electronic Science and technology and received doctor degree in engineering. In 2002-2003, he went to Russia to study at the Moscow State University as a senior visiting scholar. He is currently researching on millimeter wave, microwave technology and digital image processing technology.

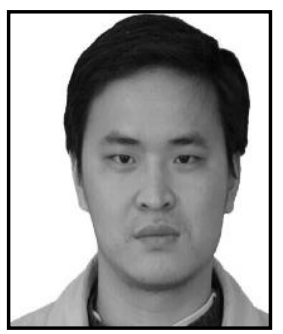

Xuegang Luo, he received the bachelor's degree in computer science and technology from Huazhong Agricultural University and the M.Eng degree in software engineering from University of Electronic Science and Technology of China. He is currently working toward the Ph.D. degree. He is currently researching on Computer Vision and Image Understanding. 